\title{
Ultraviolet downconverting phosphor for use with silicon CCD imagers
}

\author{
M. M. Blouke, M. W. Cowens, J. E. Hall, J. A. Westphal, and A. B. Christensen
}

\begin{abstract}
The properties and application of a UV downconverting phosphor (coronene) to silicon charge coupled devices are discussed. Measurements of the absorption spectrum have been extended to below $1000 \AA$, and preliminary results indicate the existence of useful response to at least $584 \AA$. The average conversion efficiency of coronene was measured to be $\sim 20 \%$ at $2537 \AA$. Imagery at $3650 \AA$ using a backside illuminated 800 $\times 800$ CCD coated with coronene is presented.
\end{abstract}

\section{Introduction}

Silicon charge coupled devices (CCD) have a number of properties that make them very attractive as scientific optical detectors, especially for space applications. Among these properties are high resolution capabilities, broad spectral range, rugged solid-state construction, geometric stability and fidelity, low readout noise, and a large dynamic range. A shortcoming of CCD detectors is their rapid loss of quantum efficiency at wavelengths shorter than $4000 \AA$.

The $800 \times 800$ pixel CCD imager has been selected for use as the detector for the Space Telescope Wide Field/Planetary Camera (ST WF/PC). Operation of the Space Telescope in earth orbit will permit astronomical observations at wavelengths that are otherwise absorbed in the atmosphere. In particular, one requirement for the detectors on the $\mathrm{ST} \mathrm{WF} / \mathrm{PC}$ is that useful response in the UV region of the spectrum down to Lyman- $\alpha$ (1216 $\AA$ ) be obtained. To achieve UV response with the CCD, a UV sensitive phosphor was selected and deposited on the active area. This paper will discuss the properties of the UV downconverting phosphor and its application to the CCD. This technique yields a detector with an extremely wide and useful optical bandwidth from the VUV into the near IR.

When this work was done A. B. Christensen was with University of Texas at Dallas; he is now with Aerospace Corporation, Los Angeles, California 90009. J. A. Westphal is with California Institute of Technology, Pasadena, California 91125. The other authors are with Texas Instruments, Inc., Dallas, Texas 75265.

Received 24 May 1980.

0003-6935/80/193318-04\$00.50/0.

(C) 1980 Optical Society of America.

\section{II. $800 \times 800 \mathrm{CCD}$}

Figure 1 presents a photograph of the $800 \times 800 \mathrm{CCD}$ imager. This device is designed for use in the thinned backside illumination mode. The construction of the device utilizes a three-phase three-level polysilicon gate technology. Pixel size is $15 \times 15 \mu \mathrm{m}$, and the membrane is $8-10 \mu \mathrm{m}$ thick. The devices are $n$-buried channel CCDs built on $10 \Omega$-cm $p$-type substrates. The devices and performance are described in detail elsewhere. ${ }^{1,2}$

\section{Coronene}

There exist a number of UV sensitive phosphors, most of which fluoresce in the spectral region near or shorter than $4000 \AA$. These are useful when used in conjunction with a photocathode or vidicon type tubes. However, the emission wavelengths of many of these phosphors do not match the useful response of silicon CCDs. One UV downconverting phosphor that does match the spectral response of silicon is coronene. ${ }^{3}$

Coronene $\left(\mathrm{C}_{24} \mathrm{H}_{12}\right)$ is a seven-ring polycyclic aromatic hydrocarbon compound based on the benzene ring. It is a yellowish powder, with a melting temperature of $360^{\circ} \mathrm{C}$, and a sublimation temperature of $\sim 200^{\circ} \mathrm{C}$ at $10^{-6}$ Torr. When excited by UV radiation of $<3800 \AA$ wavelength, coronene fluoresces in the green portion of the visible spectrum, with the peak near $5000 \AA$. This conveniently overlaps the short wavelength end of the response of the silicon CCD as shown in Fig. 2. Inokuchi et $a l .{ }^{3}$ have also shown that coronene has a constant quantum efficiency in the UV from 1400 to 3300 $\AA$.

The use of films of fluorescent materials for the detection of energetic particles and short wavelength photons is well known.,5 Figure 3 illustrates the geometry of a thin fluorescent film on a substrate (assumed to be a Si CCD) with photons incident from the film side. The possible interactions within the film are 


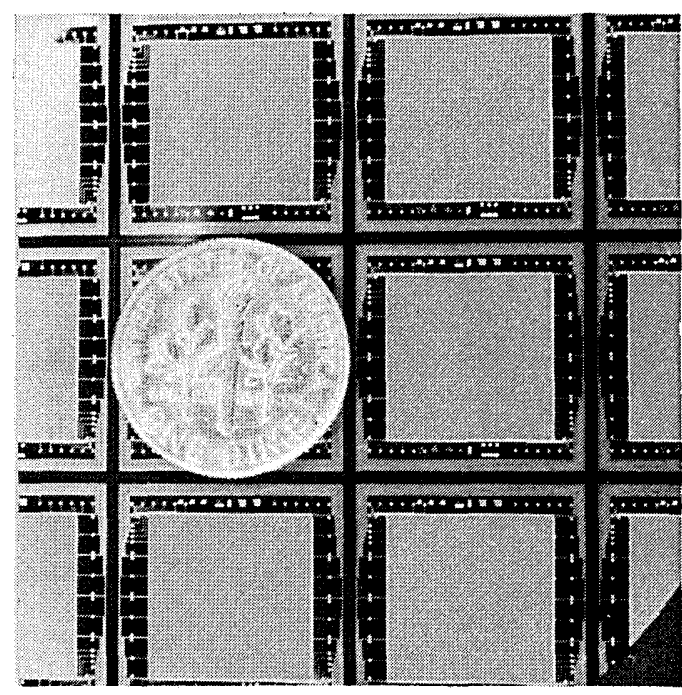

Fig. 1. Photomicrograph of $800 \times 800$ CCD Imager.

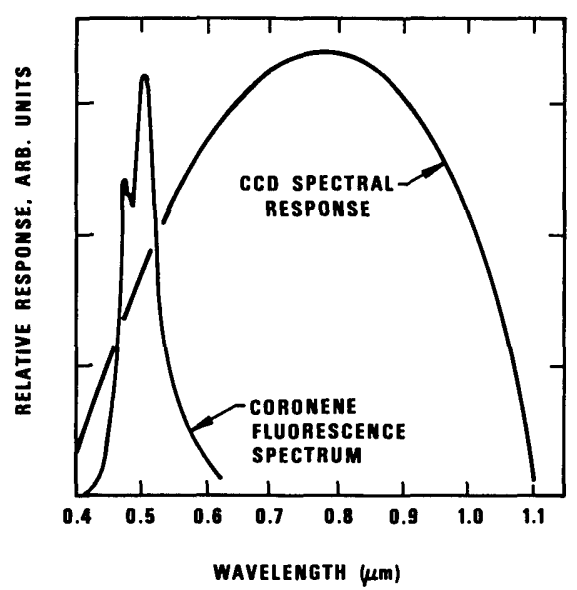

Fig. 2. Schematic diagram showing the overlap of the spectral response of a typical CCD and the fluorescence emission spectrum of coronene. $^{3}$

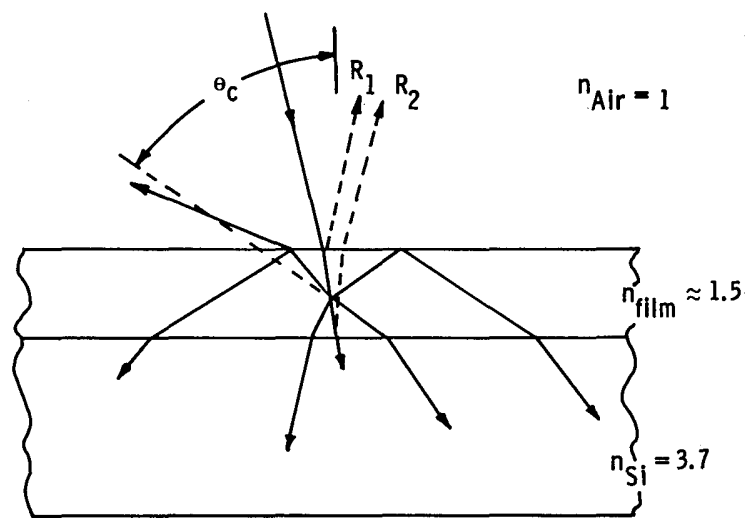

Fig. 3. Schematic representation of the geometry used to calculate the overall effective quantum efficiency of a thin phosphor layer on a silicon substrate. ${ }^{6}$ illustrated in the figure. The incident photon can be scattered and lost, reflected from either interface and lost, or absorbed within the film or semiconductor. The reemitted downconverted photon will be captured in the substrate if it is emitted at any angle greater than the critical angle $\theta_{c}$ for the film-air interface. The overall quantum efficiency of the film-silicon combination in converting UV photons to useful signal electrons in the CCD is given by the expression ${ }^{6}$

$$
\begin{array}{r}
\eta_{\mathrm{eff}}=(1-S)\left(1-R_{1}\right)\left(1-R_{2}\right) \\
\times\left\{\epsilon_{F}\left(1-F_{L}\right) A(\lambda) \bar{\eta}-[1-A(\lambda)] \eta(\lambda)\right\},
\end{array}
$$

where

$$
\begin{aligned}
& R_{1}=\left\{\frac{n_{\text {film }}-1}{n_{\text {film }}+1}\right\}^{2}, \quad R_{2}=\left\{\frac{n_{\mathrm{Si}}-n_{\text {film }}}{n_{\mathrm{Si}}+n_{\text {film }}}\right\}^{2}, \\
& F_{L}=1 / 2\left[1-\frac{\left.\left(n_{\text {film }}^{2}-1\right)^{1 / 2}\right)}{n_{\text {film }}}\right] .
\end{aligned}
$$

In these expressions $S$ is the fraction of the incident photon flux lost to scattering in the film, $R_{1}$ and $R_{2}$ are the Fresnel reflection coefficients at the air-film and film-Si interfaces, respectively, $\epsilon_{F}$ is the quantum efficiency of the phosphor film, $A(\lambda)$ is the fraction of the total incident photon flux at wavelength $\lambda$ absorbed in the film, $F_{L}$ is the fraction of the fluorescent radiation that escapes from the film, $\bar{\eta}$ is the quantum efficiency of the silicon detector averaged over the fluorescence emission spectrum of the phosphor, and $\eta(\lambda)$ is the quantum efficiency of the silicon detector at the wavelength of the incident UV photons.

Assuming $S=0, n_{\text {film }} \simeq 1.5$, and that all the UV flux is absorbed in the film,

$$
\eta_{\text {eff }}=0.76 \epsilon_{F} \bar{\eta}
$$

\section{Technique}

A number of thinned, buried channel, $800 \times 800$ chips were selected and operated as large area photodiodes. Coronene was deposited on the backside of the devices using an evaporation technique. The deposition rate varied from 1 to $>100 \AA / \mathrm{sec}$. Generally the slower rates produced more uniform appearing films. Film thickness and deposition rate were monitored with a crystal thickness monitor placed near the sample. Film thicknesses ranging from 500 to $2000 \AA$ were examined. The evaporated films are light yellow in color and have a slightly hazy appearance when viewed through a transparent substrate.

The UV measurements were performed using a 0.5 $\mathrm{m}$ Seya-Namioka grating monochromator as shown schematically in Fig. 4. The instrument is fitted with a hollow cathode discharge flowing gas UV source at the entrance slit. The devices were measured at a number of different wavelengths between 584 and $5000 \AA$, using the spectral lines emitted by gases selectively introduced into the source.

A double-beam attachment was placed at the exit slit of the instrument. A small mirror in the double-beam attachment directed the exit beam to either a calibrated photodetector or the test sample. Both an EMR photodiode, model 543P-09-00, and an EG \& G model 450 radiometer were used. The EMR detector is calibrated 
at eleven wavelengths between 1165 and $2537 \AA$, while the EG \& G unit is calibrated between 2000 and 11000 A.

Spectral response measurements of the CCD at visible and near IR wavelengths were performed using an incandescent source, $100 \AA$ wide spectral filters, and a Tektronix model J6504 probe to measure incident flux.

\section{Experimental Results}

Figure 5 presents spectral response measurements taken on a device coated with a thin film of $\sim 1600 \AA$ coronene. As may be seen from the figure, useful response to UV radiation down to $1000 \AA$ can be obtained. The overall quantum efficiency of the coronene-coated CCD at $\lambda=2537 \AA$ is $\sim 9 \%$. This is in excellent agree-

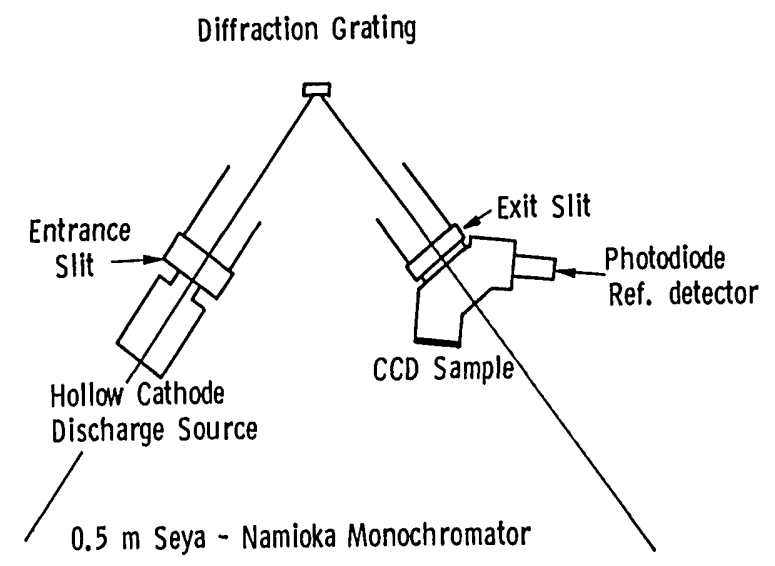

Fig. 4. Diagram of the $0.5 \mathrm{~m}$ Seya-Namioka grating monochromator. Entrance slit is differentially pumped to permit higher pressure op-' eration of the gas discharge source.

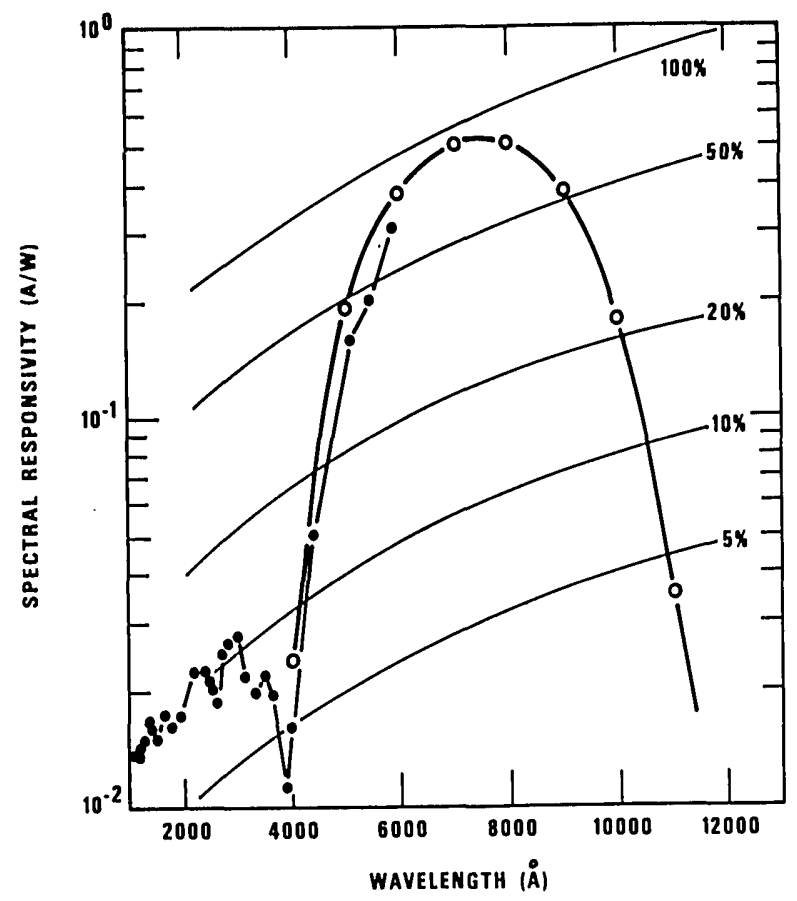

Fig. 5. Spectral response curve of a CCD with $\sim 1600 \AA$ of coronene. Also presented are theoretical curves of response at constant quantum efficiency.

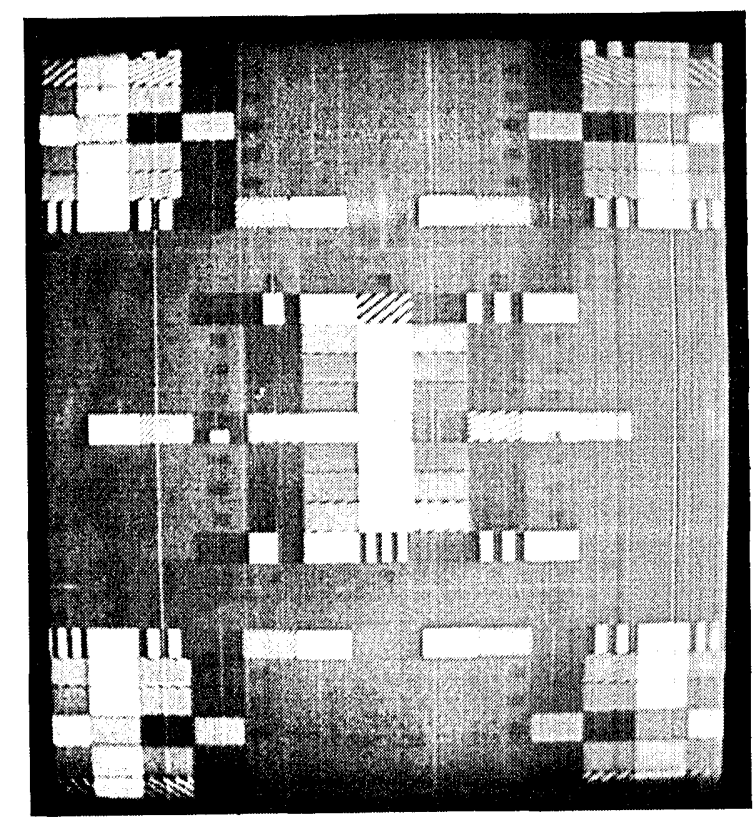

Fig. 6. Imagery with a thinned backside illuminated $800 \times 800 \mathrm{CCD}$ coated with $1300 \AA$ of coronene.

ment with the predictions of Eq. (2) using the measured value of $\epsilon_{F}(2537 \AA) \simeq 20 \%$ and a calculated value of $\bar{\eta}$ $\simeq 1 / 2$ for this device. ${ }^{7}$

A dip in the phosphor/CCD response exists in the neighborhood of $3800 \AA$. This dip results from the fact that the fluorescent efficiency of coronene decreases rapidly near $3800 \AA$, while the CCDs do not exhibit any significant response until $\sim 4000 \AA$.

Imaging properties of a thinned $800 \times 800$ device coated with $1300 \AA$ of coronene are presented in Fig. 6 . The target is the RCA slant bar chart, illuminated with a strobed source, and filtered with a narrow-pass filter centered at $3650 \AA$. Perusal of the figure shows that 800 -lines/picture height can be discerned. This represents the Nyquist frequency of the device and indicates that the device/coating have good spatial resolution in the UV.

Modulation transfer function (MTF) data for this device have been taken at selected wavelengths between 4000 and $8000 \AA$. These data are presented in Fig. 7 and are corrected for the lens MTF. Note that the CCD $\mathrm{MTF}$ at $\lambda=4000 \AA$ is $\simeq 43 \%$ at the spatial Nyquist frequency ( 33 cycles $/ \mathrm{mm}$ for this device). At the longer wavelengths the MTF is even better, as would be expected. 8 These data clearly demonstrate that the deposited coronene film does not adversely affect the spatial resolution of the device in the visible region of the spectrum.

As a serendipity, thin layers of coronene $(\$ 2000 \AA)$ appear to act as antireflection coatings, enhancing the response of the coated device throughout the visible and near IR spectral regions of normal CCD operation. This effect is illustrated in Fig. 8, where spectral response is presented before and after coating with 1600 $\AA$ of coronene. The effect has not been investigated for layers $>2500 \AA$. 


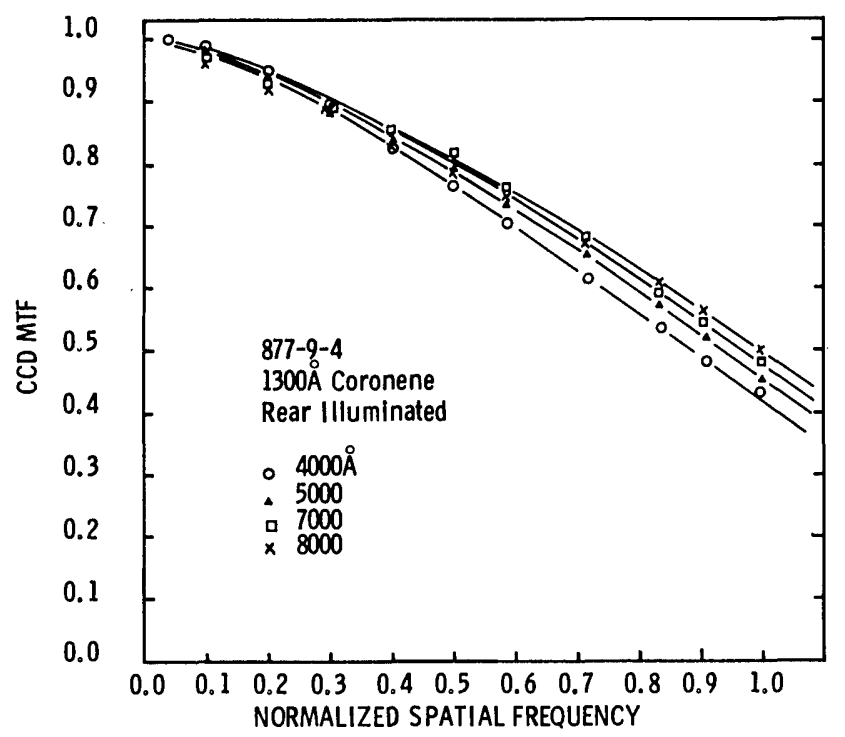

Fig. 7. Modulation transfer function data for the device of Fig. 6. Spatial Nyquist frequency for this device is 33 cycles $/ \mathrm{mm}$.

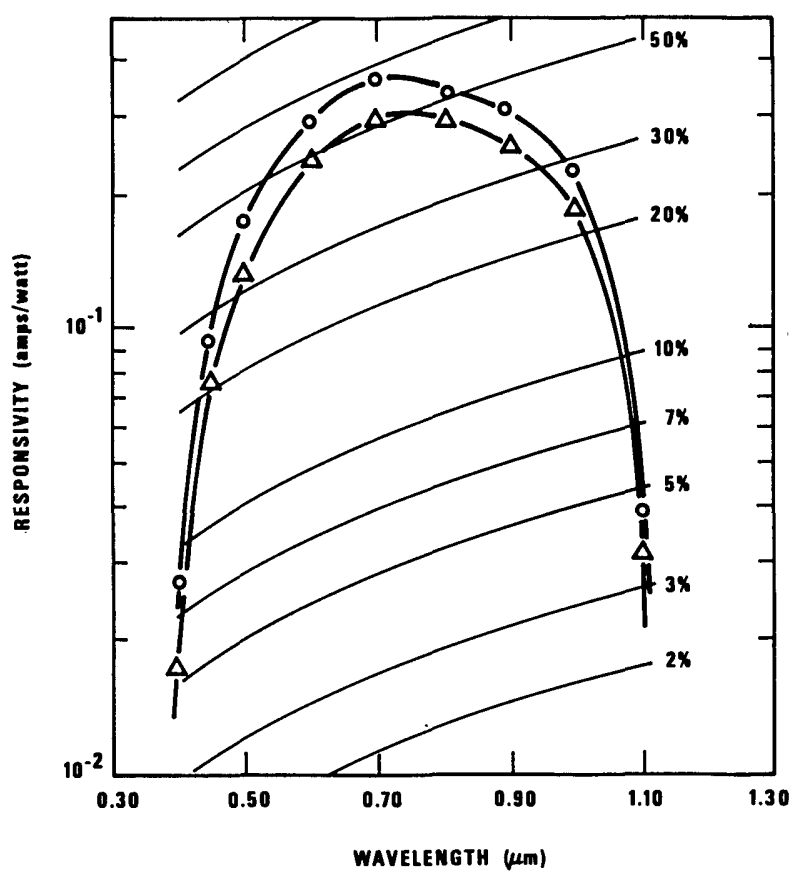

Fig. 8. Spectral response data before and after coating with $1600 \AA$ of coronene, showing the enhancement in visible and near IR response.

Absorption coefficients have been measured at 2500 and $3600 \AA$. These data suggest an $\alpha$ of $\sim 1.5-3.0 \times 10^{5}$ $\mathrm{cm}^{-1}$. This implies that a thin layer of coronene between 1000 and $2000 \AA$ should be adequate to absorb almost all the UV radiation incident on the device.

\section{Summary}

A technique has been developed to deposit a UV downconverting phosphor, coronene, on a thinned backside illuminated CCD. Spectral response measurements show that the coronene extends the useful response of the CCD down to at least $1000 \AA$ in the
VUV. Preliminary measurements show response to at least $584 \AA$. The coronene also tends to act as an antireflection coating, enhancing the response of the coated device throughout the visible and near IR spectral regions of normal CCD operation. It is clear that the use of this technique is not restricted to CCDs.

The authors would like to express their appreciation for the efforts of L. Anschutz, J. Wilson, and M. Miles during the course of this work and to $\mathrm{H}$. H. Hosack.

The work described in this paper is supported by the National Aeronautics and Space Administration under NASA contract NAS 7-100 and was performed for the Jet Propulsion Laboratory, California Institute of Technology.

\section{References}

1. M. M. Blouke, J. E. Hall, and J. F. Breitzmann, "A 640,000 pixel CCD Imager for Space Applications," in Proceedings, 1978 IEDM, Washington, D.C. (IEEE, Dec. 1978).

2. F. P. Landauer, J. R. Janesick, S. L. Knapp, M. M. Blouke, and J. E. Hall, "An $800 \times 800$ CCD Imager for Space-Borne Scientific Imaging," in Proceedings, 1978 GOMAC, Monterey, Calif. (Nov. 1978).

3. H. Inokuchi, K. Harada, and T. Kondow, J. Opt. Soc. Am. 54, 842 (1964).

4. J. B. Birks, The Theory and Practice of Scintillation Counting (Pergamon, London, 1964).

5. J. A. R. Samson, Techniques of Vacuum Ultraviolet Spectroscopy (Wiley, New York, 1967).

6. W. Viehmann, "Thin Film Scintillators for Extended UV-Response Silicon Detectors," Proc. Soc. Photo-Opt. Instrum. Eng. 196, (1979).

7. A. B. Christensen, M. W. Cowens, and M. M. Blouke, "Coronene Ultraviolet Fluorescence," in preparation.

8. D. E. Seib, IEEE Trans. Electron Devices ED-21, 217 (1974). 